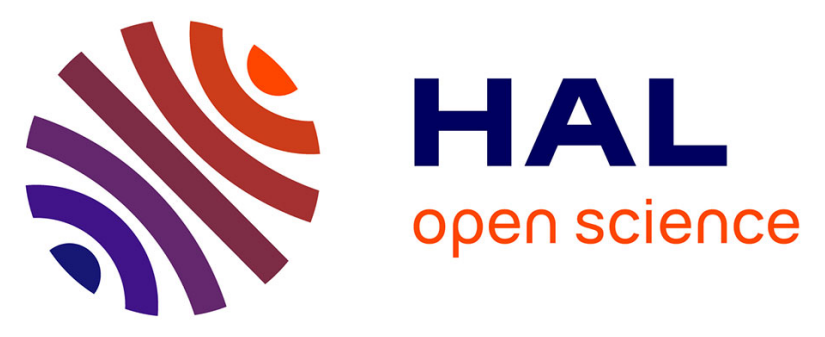

\title{
Chauffage par effet plasmon pour la désorption localisée de cellules capturées sur une biopuce
}

\author{
Elodie Engel, Radoslaw Bombera, Loic Leroy, Roberto Calemczuk, Loic \\ Laplatine, Dieudonné Baganizi, Patrice N. Marche, Yoann Roupioz, Thierry \\ Livache
}

\section{To cite this version:}

Elodie Engel, Radoslaw Bombera, Loic Leroy, Roberto Calemczuk, Loic Laplatine, et al.. Chauffage par effet plasmon pour la désorption localisée de cellules capturées sur une biopuce. 7ème Colloque Interdisciplinaire en Instrumentation, Jan 2016, Saint-Nazaire, France. hal-01280220

\section{HAL Id: hal-01280220 \\ https://hal.science/hal-01280220}

Submitted on 29 Feb 2016

HAL is a multi-disciplinary open access archive for the deposit and dissemination of scientific research documents, whether they are published or not. The documents may come from teaching and research institutions in France or abroad, or from public or private research centers.
L'archive ouverte pluridisciplinaire HAL, est destinée au dépôt et à la diffusion de documents scientifiques de niveau recherche, publiés ou non, émanant des établissements d'enseignement et de recherche français ou étrangers, des laboratoires publics ou privés. 


\title{
Chauffage par effet plasmon pour la désorption localisée de cellules capturées sur une biopuce
}

Elodie Engel1,2,3, Radoslaw Bombera ${ }^{1,2,3}$, Loïc Leroy 1,2,3, Roberto Calemczuk ${ }^{1,2,3}$, Loïc Laplatine ${ }^{1,2,3}$, Dieudonné R. Baganizi ${ }^{1,2,3}$, Patrice N. Marche $^{4}$, Yoann Roupioz ${ }^{1,2,3}$ et Thierry Livache ${ }^{1,2,3}$

${ }^{1}$ Univ. Grenoble Alpes, INAC-SPrAM, F-38000 Grenoble, France ${ }^{2}$ CNRS, SPrAM, F-38000 Grenoble, France ${ }^{3}$ CEA, INAC-SPraM, F-38000 Grenoble, France

4 Institut Albert Bonniot INSERM-UJF U823 Grenoble France

elodie.engel@ujf-grenoble.fr

\begin{abstract}
Photo-thermal Effect for Localized Desorption of Primary Lymphocytes Arrayed on an Antibody/DNA-based Biochip)
\end{abstract}

This work proposes a miniaturized system able to perform multiple cell capture followed by cell-type selective release from a biochip surface. Unlabeled lymphocytes are first specifically captured onto a DNA array by antibody-DNA conjugates. The immobilized cells are subsequently released under spatiotemporal control within local heating generated by intense Surface Plasmon Resonance (SPR) produced by laser illumination.

\section{Introduction}

L'examendétaillé d'échantillons biologiques complexes comme le sang, présente un grand intérêt pour larecherche en biologie, les biotechnologies ou les applications biomédicales[1,2]. Analyser de façon pertinente qualitativement et quantitativement un milieu complexe nécessite de pouvoir extraire des sous-populations de cellules à partir d'un mélange hétérogène [3].Assurer un tel tri multiplexé dans des conditions compatibles avec la survie cellulaire représente un défi ambitieux. II existe des dispositifs micro-structurés et micro-fluidiques capables de séparer des cellules par l'intermédiaire de contraintes mécaniques [4], de reconnaissance antigène/anticorps [5], d'application d'un champ magnétique [6] etc. Cependant, ces techniques sont limitées en termes de multiplexage et requièrent souvent un marquage préliminaire des échantillons (photo- or magneto-marquage pour les systèmes $\mu$ FACS ou $\mu \mathrm{MACS}$ ). C'est pourquoi il est intéressant de concevoir des micro-systèmes basés sur des surfaces " bio-sensibles » capables de faire du tri cellulaire multiplexé et sans marquage [7]. L'objectif de ces micro-systèmes est de capturer des cellules ciblées et de procéder à leur relargage sélectif et contrôlé sans altérer leur survie. Dans les dispositifs existant, le relargage est contrôlé mécaniquement [8], électriquement $[9,10]$, thermiquement [11] ou par des enzymes [12,13]. Notre équipe a quant à elle développé un système de tri cellulaire sans marquage basé sur les propriétés thermodynamiques de l'ADN et sur la résonance des plasmons de surface (SPR) [14]. 


\section{Principe et méthodes}

\subsection{Principe physique de l'expérience}

Les cellules ou les molécules ciblées sont capturées sur la surface dorée d'une biopuce grâce à des anticorps spécifiques conjugués à des brins d'ADN (Figure 1). Ces brins d'ADN (conjugués aux anticorps) sont hybridés à leur brin complémentaire qui est immobilisé sur la surface dorée de la biopuce. Un chauffage laser localisé induit la déshybridation des doubles brins d'ADN et entraîne la libération des molécules/cellulescapturées.

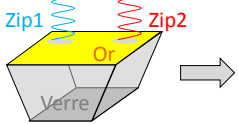

1)

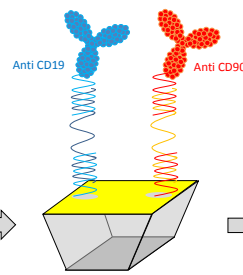

2)

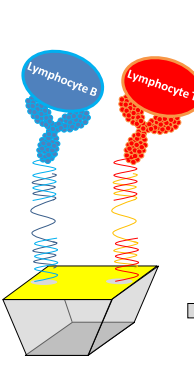

3)

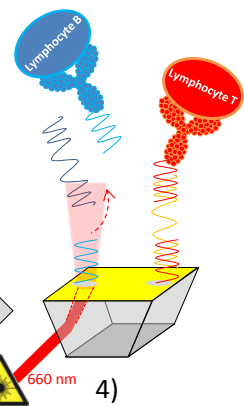

Figure 1: Principe de la stratégie de capture/relargage des cellules. 1) Greffage des ADN sur la surface d'or. 2) Hybridation des ADN complémentaires conjugués à des anticorps anti-lymphocytes.3) Immobilisation des lymphocytes B et T. 4) Désorption localisées des cellules par chauffage laser.

Le chauffage est réalisé par le dessous de l'échantillon. Dans cette configuration, l'échantillon n'est pas exposé à la totalité du flux de lumière incident mais seulement à l'onde évanescente. Dans ces conditions, le chauffage est localisé à l'interface or/milieu ce qui limite l'exposition du milieu à la chaleur et assure un impact minimal sur les échantillons biologiques libérés. Ce dernier point est essentiel étant donné que notre objectif final est le tri de cellules sanguines et que la plupart des cellules de mammifère sont très sensibles au stress thermique [15].

\subsection{Dispositif}

Notre stratégie repose sur une désorption physique induite par un chauffage localisé généré par un rayonnement laser continu appliqué dans les conditions de résonance des plasmons de surface (SPR) [16]. Le dispositif est basé sur une configuration optique représentée Figure 2. II est dépourvu d'éléments de chauffage sophistiqués [17] et assure une élévation localisée de température. Ce dispositif, conçu au laboratoire, est constitué d'un système d'imagerie SPR classique(Figure 2a) complété parun microscope (pour permettre des observations par le dessus de la puce) et d'une source laser (qui induit le chauffage localisé)(Figure 2b). Le dispositif laser convergent, polarisé transverse magnétique et de puissance ajustable $(\lambda=660 \mathrm{~nm} P=500 \mathrm{~mW})$ est monté sur platine $x / y$ qui permet de choisir la zone de la biopuce sur laquelle induire l'effet photothermique recherché. Sous l'ange d'incidence approprié, l'énergie des photons incidents est en premier lieu partiellement convertie en oscillation collective des électrons (plasmon de surface) puis finalement dissipée 
Chauffage par effet plasmon pour la désorption localisée de cellules capturées sur une biopuce

en chaleur dans le milieu environnant. La puissance laser effective absorbée par couplage avec les plasmons de surface est de l'ordre de $1 \mu \mathrm{W} / \mu \mathrm{m}^{2}$.

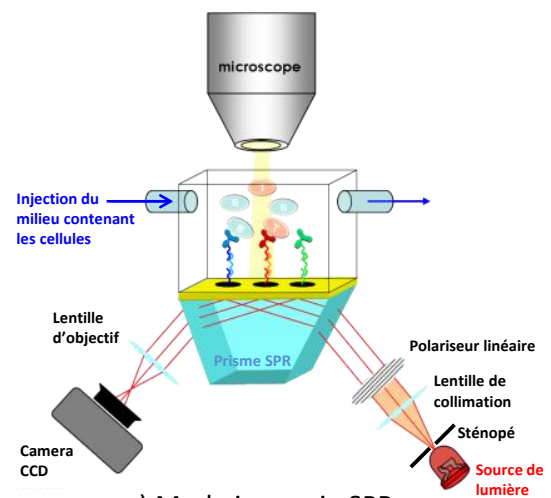

a) Mode imagerie SPR

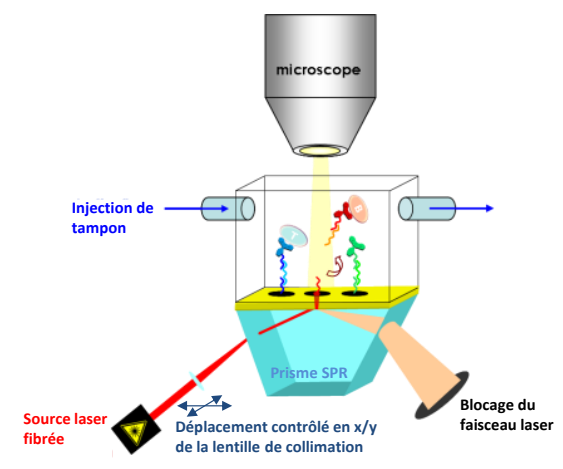

b) Mode chauffage laser

Figure 2 :Schéma du dispositifexpérimental utilisé dans les expériences de désorption induite par laser. a) Mode imagerie SPR (SPRi) : dispositif standard de SPRi complété avec un microscope qui permet le contrôle en temps réel de la construction de l'assemblage moléculaire sur la biopuce(par SPRi )et des étapes de capture et de relargage des cellules(par microscopie). b) Mode laser : configuration optique pour le chauffage SPR localisé permettant la désorption photo-thermique des espèces capturées sous l'effet du rayon laser.

\subsection{Protocoles}

La libération des cellules dépend des propriétés thermosensibles des doubles brins d'ADN qui sous l'effet du chauffage se déshybrident et entraînent le relargage contrôlé des objets ciblés (ici des lymphocytes). En premier lieu, des assemblages moléculaires assurent la capture spécifique de cellules sur une puce à $A D N$. Les expériences ont été réalisées sur un prisme doré fonctionnalisé par électropolymérisation [18] avec de courtes séquences d'ADN, les oligonucléotides Zip1, Zip2 et Zip3. Le réseau de sondes d'ADN était composé de plusieurs plots de $1 \mathrm{~mm}$ de diamètre. La puce a ensuite été assemblée dans un système micro-fluidique intégré au dispositif SPRi et convertie en puce à anticorps capables de réaliser des captures spécifiques de cellules par l'intermédiaire d'anticorps conjugués à de I'ADN [12]. Les cellules ont ensuite été injectées sur la puce grâce au circuit micro-fluidique. L'assemblage moléculaire a été érigé à l'aide de brins d'ADN intermédiaires (Zip1*-Zipl, Zip2*-Zipll, Zip3*-ZipllI où Zip1* ${ }^{*}$ Zip2* et Zip3* correspondent respectivement aux séquences complémentaires de Zip1, Zip2 et Zip3) et complété par des anticorps liés covalemmentà des ADN complémentaires des brins intermédiaires (Zipl ${ }^{*}$ Zipl|*, ZiplII*) [12].

\section{Résultats}

La faisabilité de la désorption a tout d'abord été démontrée sur l'assemblage moléculaire (ADN/ADN intermédiaire/ADN complémentaire-anticorps). Des anticorps modèle (IgG de lapin) ont été utilisés et incorporés sur la biopuce dans cet objectif. L'imagerie par SPR a permis la visualisation en temps réel des étapes de construction de l'assemblage moléculaire 
sur la puce (Figure 3a). Une fois les assemblages moléculaires ADN-lgG terminés, la désorption spécifique de certaines sondes a été induite par chauffage laser. Le relargage a été réalisé en concentrant le faisceau laser sur une zone ciblée de la biopuce. Afin de réduire la température de dissociation des ADN double brins et d'assurer une dénaturation efficace des substrats, nous avons utilisé un tampon composé de PBS (Phosphate salin buffer) comprenant $20 \%$ (v/v) d'agent déstabilisant N,N-dimethylformamide (DMF). Le DMF permet d'abaisser la température de dissociation des ADN double-brin d'en moyenne0.58 \pm 0.05 ${ }^{\circ} \mathrm{C} / \% \mathrm{DMF}[19]$. Nous avons vérifié qu'il n'affectait pas la survie cellulaire. La libération des anticorps a été réalisée pour les sondes (Zip2 et Zip3) en focalisant la source laser sur les plots correspondant à la surface de la biopuce. Deux trajectoires du laser ont été enregistrées sur la surface de la biopuce. On observe une diminution de la réflectivité correspondant au relargage adéquat des composants ciblés. Cette observation est confirmée par l'image SPR qui révèle les zones où le matériel biologique a été enlevé (Figure $3 b$ ). Une fois la désorption terminée, de l'ADN complémentaire correspondant au matériel désorbé a été réintroduit dans le système et correctement ré-hybridé. Les architectures moléculaires ont donc été restaurées ainsi que le signal SPR, prouvant ainsi l'intégrité et la capacité de régénération de la biopuce.

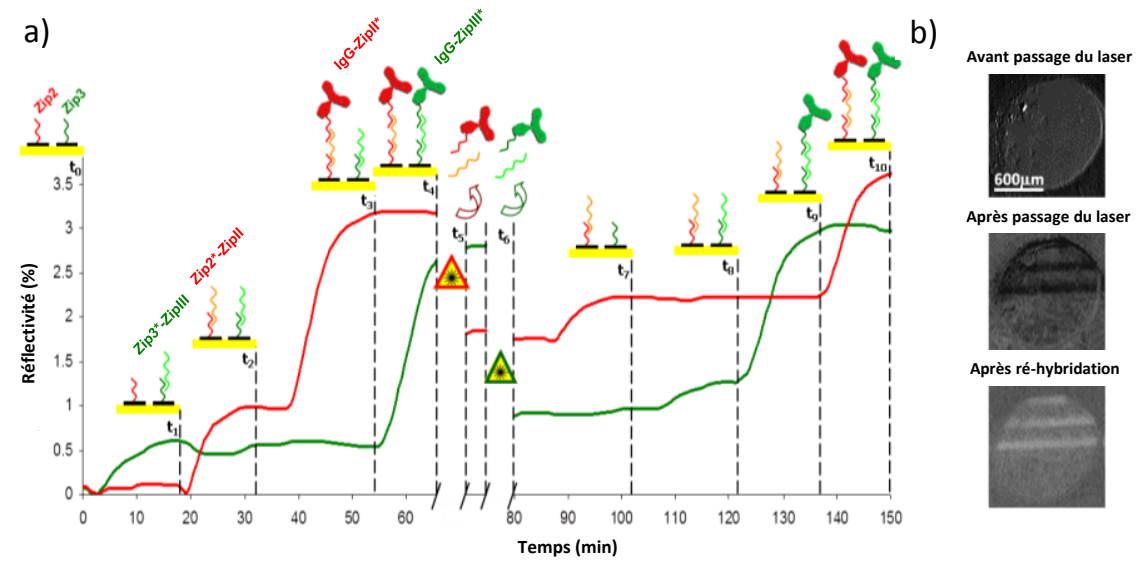

Figure 3 : Contrôle en temps réel de la construction moléculaire de la biopuce et de la désorption induite par chauffage laser localisé. a) Réflectivité enregistrée pour les sondes Zip2 et Zip3 : to - puce originale à $A D N$ (Zip2 et Zip3); $t_{1}, t_{2}$ - hybridation des brins intermédiaires ; $t_{3}, t_{4}$ - hybridation des anticorps conjugués à l'ADN ; t5 - désorption de Zip2 ; t6 - désorption de Zip3; t7-t10 - re-hybridation des brins complémentaires conjugués aux anticorps. b) Image SPR d'un plot d'ADN avant et après désorption laser suivie de l'étape de ré-hybridation.

Les résultats précédents ayant validé le concept de la désorption localisée des assemblages moléculaires, nous avons ensuite testé la désorption de cellules vivantes. Des splénocytes de souris, contenant à la fois des lymphocytes $B$ et $T$, ont été immobilisés à la surface de la biopuce grâce à des anticorps spécifiques [3]: anti-CD19 et anti-CD90 respectivement. Ces anticorps ont été au préalable conjugués avec les oligonucléotides Zipl* et ZiplI* (respectivement complémentaires des brins intermédiaires Zipl et Zipll) puis immobilisés sur les plots Zip1 et Zip2. L'oligonucléotide Ziplll* (complémentaire de Ziplli) a été conjugué avec un anticorps anti-HcB incapable de reconnaitre les cellules $B$ et $T$ et a servi de contrôle négatif. La suspension de splénocytes a été injectée dans le circuit de 
Chauffage par effet plasmon pour la désorption localisée de cellules capturées sur une biopuce

micro-fluidique et mis en contact avec la puce à anticorps. L'immobilisation des cellules a été imagée par microscopie (Figure 4, to). L'introduction de la suspension cellulaire a entraîné une capture efficace des lymphocytes B et T sur les plots Zip1 et Zip2 respectivement ( 300 cellules/plot) et pas de capture significative sur le plot contrôle Zip3. Après immobilisation des lymphocytes sur la puce, nous avons procédé au détachement des cellules par illumination laser et sous flux. La désorption photo-thermique a été induite localement sur chaque plot et suivie en temps réel par microscopie (Figure $4 t_{1}$ à $t_{3}$ ). Les images suggèrent que le passage du laser induit un détachement instantané des cellules. Le contrôle à distance de la trajectoire du faisceau laser permet les relargages successifs et indépendants des lymphocytes $B$ et $T$. Le taux de détachement a été estimé à $89 \pm 6 \%$. Finalement, la biopuce a été régénérée avec succès et des cellules spécifiques ont à nouveau été efficacement capturées. La seconde capture, réalisée après ré-hybridation, montre que le chauffage induit seulement l'ouverture de l'ADN double brin et que le système de tri cellulaire proposé est réutilisable (Figure $\left.4, t_{4}\right)$.

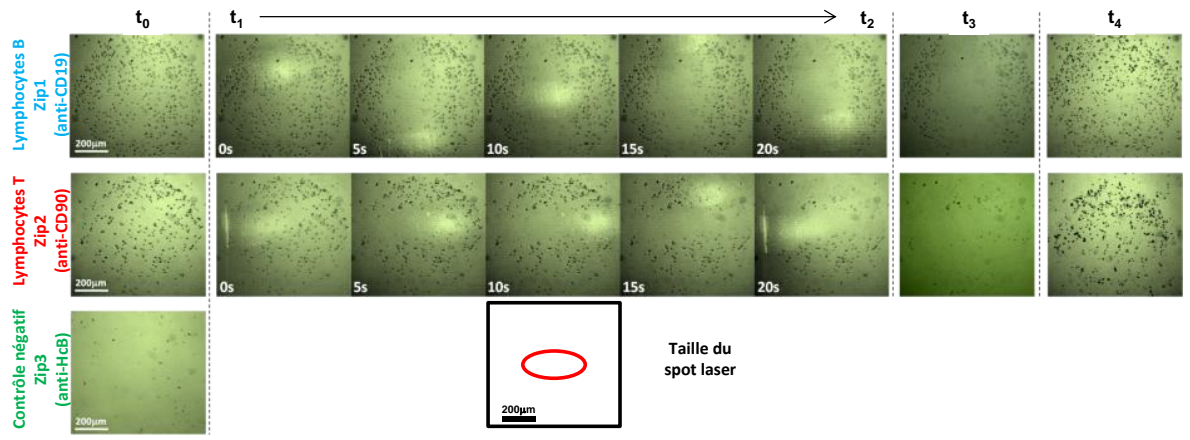

Figure 4 : Désorption localisée de lymphocytes par chauffage local. to) image au microscope de cellules capturées sur leurs plots spécifiques ADN/anticorps ; $t_{1}, t_{2}$ ) relargage localisé des cellules durant le passage du laser ; $t_{3}$ ) plots ADN/anticorps observés après le passage du laser; $t_{4}$ ) réhybridation de cellules sur leurs plots " sonde » spécifiques après reconstruction de l'assemblage moléculaire.

Afin de déterminer la température à laquelle étaient exposées les cellules lors du chauffage, nous avons mesuré les températures de dissociation des doubles brins utilisés pour la capture cellulaire : Zip1/Zip1* et Zip2/Zip2*. Les courbes de dénaturation (Figure 5) indiquent une température de dissociation égale à $63 \pm 3{ }^{\circ} \mathrm{C}$. La température à laquelle sont exposées les cellules est très probablement équivalente. Cependant le détachement est rapide, grâce au flux les cellules restent exposées moins de 3 secondes (estimation effectuée par l'analyse des séquences des désorptions). 


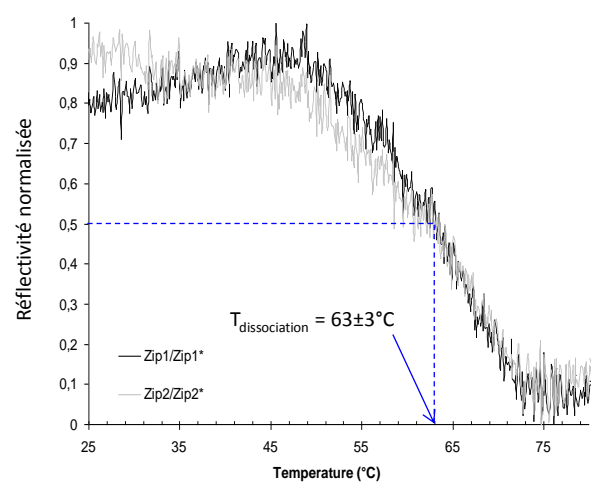

Figure 5 : Courbes de dénaturation pour Zip1/Zip1* et Zip2/Zip2* obtenues dans du PBS 20\% DMF.

Afin d'estimer l'impact sur les cellules de la températureinduite par le chauffage laser, nous avons exposé des splénocytes à $65^{\circ} \mathrm{C}$ pendant 3 secondes. Nous avons ensuite mesuré les proportions de cellules vivantes dans les échantillons chauffés et non chauffés. On constante que le taux de (sur)mortalité dû au chauffage n'excède pas 16\% (Figure 6). Pour des développements futurs, d'autres solutions pourraient être envisagées pour réduire la température à laquelle sont exposées les cellules. Par exemple, nous pourrions utiliser un laser pulsé afin d'augmenter le gradient de température entre la surface et les cellules. Nous pourrions également utiliser des séquences d'ADN présentant des défauts d'appariement et donc des températures de dissociation plus faibles.

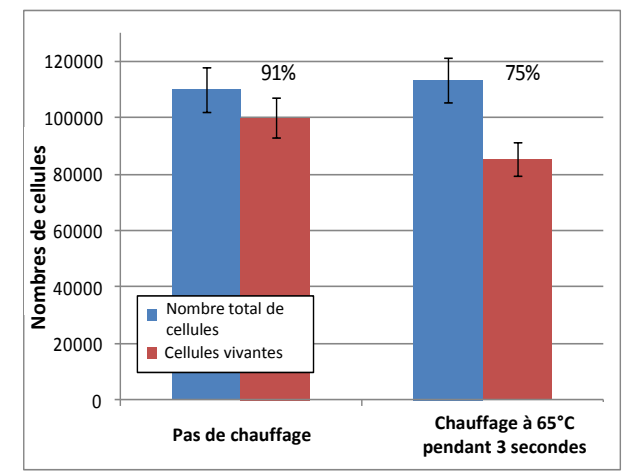

Figure 6: Comptages cellulaires et mesures de viabilité sur les échantillons chauffés et non chauffés.

\section{Conclusion}

Nous avons développé une micro-plateforme capable de réaliser la capture multiplexée de cellules vivantes spécifiques et leur désorption contrôlée. Ce systèmeest basé sur la conjugaison d'anticorps et de brin d'ADN et tire profit des propriétés thermodynamiques de I'ADN. Cette approche innovante constitue une méthode précise, économe (en réactif) et non 
Chauffage par effet plasmon pour la désorption localisée de cellules capturées sur une biopuce

invasive pour le tri cellulaire multiplexé. Dans l'avenir, cette biopuce pourrait être adaptée pour capturer et relâcher plus de deux types cellulaires et permettre l'analyse d'échantillons biologiques bruts tels que le sang. Cela nécessiterait l'intégration d'acides nucléiques stabilisés chimiquement afin d'améliorer la résistance de l'ADN aux nucléases présentes dans le sang. Ces modifications n'engendreraient pas de changements significatifs des propriétés thermodynamique de la molécule. Notre approche pourrait alors être utilisée pour étudier par exemple des phénomènes immunologiques complexes : les cellules, tout d'abord identifiées et triées, pourraient être individuellement détachées pour des analyses ultérieures telles que le séquençage d'ADN ou d'ARN.

\section{Remerciements}

Les auteurs remercient l'Agence Nationale pour la Recherche pour le financement de ce projet (Multicells, ANR-BLANC-1519-01) ainsi que la fondation Nanosciences RTRA pour le financement de la thèse de R.B. Ce travail a également été soutenu par le Labex ARCANE (ANR-11-LABX-0003-01).

\section{Bibliographie}

[1] Liotta LA, Ferrari M, Petricoin E. Clinical proteomics: Written in blood. Nature.2003, 425, 905.doi:10.1038/425905a

[2] Anderson NL, Anderson NG. The human plasma proteome: history, character, and diagnostic prospects. Mol. Cell. Proteomics. 2002, 1, 845

[3] Roupioz Y, Berthet-Duroure N, Leïchlé T, Pourciel JB, Mailley P, Cortes S, Villiers MB, Marche PN, Livache T, Nicu L. Individual Blood-Cell Capture and 2D Organization on Microarrays. Small. 2009, 5, 1493

[4] Kim SM, Lee SH, Suh KY. Cell research with physically modified microfluidic channels: a review. Lab Chip. 2008, 8, 1015

[5] Liu J, Bombera R, Leroy L, Roupioz Y, Baganizi DR, Marche PN, Haguet V, Mailley P, Livache T, Selective individual primary Cell capture Using locally bio-Functionalized Micropores, Plos One. 2013, 8, DOI 10.1371/journal.pone.0075717

[6] Adams JD, Kim U, Soh HT, Multitarget magnetic activated cell sorter. Proc. Natl. Acad. Sci. U. S. A. 2008, 105, 18165

[7] Didar TF, Tabrizian M. Adhesion based detection, sorting and enrichment of cells in microfluidic Lab-on-Chip devices. Lab Chip. 2010, 10, 3043

[8] Wang Y, Phillips C, Xu W, Pai JH, Dhopeshwarkar R, Sims CE, Allbritton N. Micromolded arrays for separation of adherent cells. Lab Chip.2010, 10, 2917.doi: 10.1039/c0lc00186d

[9] Zhu H, Yan J, Revzin A, Catch and release cell sorting: electrochemical desorption of T-cells from antibody-modified microelectrodes. Colloids Surf B Biointerfaces.2008, 64, 260.doi: 10.1016/j.colsurfb.2008.02.010.

[10] Yeo WS, Mrksich M. Electroactive Self-Assembled Monolayers that Permit Orthogonal Control over the Adhesion of Cells to Patterned Substrates. Langmuir, 2006, 22, 10816.

[11] Hatakeyama H, Kikuchi A, Yamato M, Okano T. Patterned biofunctional designs of thermoresponsive surfaces for spatiotemporally controlled cell adhesion, growth, and thermally induced detachment. Biomaterials.2007, 28, 3632. 
[12] Bombera R, Leroy L, Livache T, Roupioz Y. DNA-directed capture of primary cells from a complex mixture and controlled orthogonal release monitored by SPR imaging. Biosensors and Bioelectronics.2012, 33, 10. doi.org/10.1007/978-1-61779-373-8_5

[13] Kwong GA, Radu CG, Hwang K, Shu CJY., Ma C, Koya RC, Comin-Anduix B, Hadrup SR, Bailey RC, Witte ON, Schumacher TN, Ribas A and Heath JR. Modular nucleic acid assembled p/MHC microarrays for multiplexed sorting of antigen-specific T cells. J Am Chem Soc. 2009, 131, 9695. doi: 10.1021/ja9006707.

[14] Leroy L, Bombera R, Engel E, Calemczuk R, Laplatine L, Baganizi DR, Marche PN, Roupioz Y, Livache T. Photo-thermal Effect for Localized Desorption of Primary Lymphocytes Arrayed on an Antibody/DNA-based Biochip. Lab Chip. 2014, 14, 1987

[15] Chen Q, Fisher DT, Kucinska SA, Wang WC, Evans SS. Dynamic control of lymphocyte trafficking by fever-range thermal stress. Cancer Immunollmmunother, 2006, 55, 299

[16] Xiao XJ, Gao Y, Xiang JA, Zhou FM. Laser-Induced Thermal Effect in Surface Plamson Resonance. Anal.Chim.Acta, 2010, 676, 75

[17] Kajiyama T, Miyahara Y, Kricka LJ, Wilding P, Graves DJ, Surrey S, Fortina P. Genotyping on a thermal gradient DNA chip. Genome Res. 2003, 13, 467

[18] Fuchs J, Fiche JB, Buhot A, Calemczuk R, Livache T. Salt concentration effects on equilibrium melting curves from DNA microarrays. Biophys J. 2010, 99, 1886.doi: 10.1016/j.bpj.2010.07.002

[19] Bressan G, Rampone R, Bianchi E, Ciferri A. DNA conformation in N,N-dimethyl formamide$\mathrm{H}_{2} \mathrm{O}$ solutions. Biopolymers, 1974, 13, 2227 